\title{
SECURE ENCRYPTION ROUTING FOR MANET
}

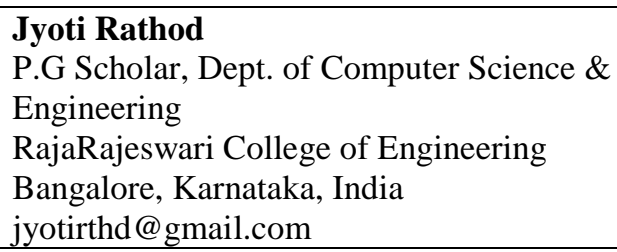

\author{
K.S. Rajesh \\ Associate Prof. Dept of Computer Science \& \\ Engineering \\ RajaRajeswari College of Engineering \\ Bangalore, Karnataka, India \\ rajeshks_hrr@yahoo.com
}

\begin{abstract}
Mobile Adhoc network are self configuring infrastructure less network. In MANET we use a new anonymous routing protocol which hides the nodes identity and also the route. The earlier schemes provide anonymity, unlinkability and location anonymity. One of the main objectives is unobservability and Undetectability. The unobservablilty makes the packet content safe so that the data is not modified by the different entities. The Undetectability means the attacker cannot detect who is sending data to whom and whether the data exists or not. The routing protocol used here is onion routing which protects the intermediates nodes from inferring the destination and uses the route secret verification message by using pseudonyms. Group keys are used for the secure anonymous encryption in MANET.
\end{abstract}

Index Terms - Anonymous, Mobile Adhoc network, Pseudonymity Routing protocol, Security.

\section{INTRODUCTION}

The Manet is a Mobile Adhoc Network which is self configuring network. It does not have any fixed infrastructure or connectivity. Therefore it is free to move with in a particular region. Adhoc network are different from other networks. The reasons for this is it can be reconfigured any time, it has low bandwidth compared to wired network, it can also be redeployed at any place which is difficult in wired networks and it also consists of high mobility. The adhoc network fall under three main categories. They are Reactive routing protocol, Proactive routing protocol and the last one is Hybrid routing protocol which is combination of Reactive routing protocol and Proactive routing protocol. The Mobile Adhoc Network can be used in many applications such as Military communication, file sharing, online voting, and underwater rescue operation.
Anonymous routing protocol means the nodes in the network are anonymous to each other. The reasons for using anonymous routing protocol are the security, which is one of the hot issues in mobile adhoc networks. Providing security to the data and the path through which the data is to be transferred is one of the big challenges in today's environment. The existing anonymity routing protocol can be categorized into two categories. The first is hop-by-hop encryption and the second is redundant traffic. We use hop-by-hop encryption method where the hop-by-hop encryption method is again divided into two methods. They are onion routing and hop-by-hop authentication. We use both onion routing and hop-by-hop authentication for routing the packet from source to the destination to provide security and authenticity. The second method is redundant traffic. The traffic is the major concerned in order to deliver the packet from source to the destination.

The onion routing protocol is one of the effective routing protocol which makes the data or packet content safe. So that the intruders cannot hack the data. The data and the node identities are not revealed. Instead of the real node identities node pseudonyms are used to transfer the content of data from one node to other node.

\section{LITERATURE SURVEY}

Here we describe how the earlier protocols were used to transfer the data from one node to the other node. 


\section{A. Unobservable Privacy-preserving Routing in MANET}

P. Thamizharasi, D.Vinoth (2013) proposed the Unobservable Privacy-Preserving Routing protocol [3]. An obvious drawback in existing schemes is that packets are not protected as a whole. An efficient privacy-preserving routing protocol USOR that achieves content unobservability by employing anonymous key establishment based on group signature. USOR is to protect all parts of a packet's content and it is independent of solutions on traffic pattern unobservability. The unobservable routing protocol is then executed in two phases. First, an anonymous key establishment process is performed to construct secret session keys. Finally the unobservable route discovery process is executed to find a route to the destination to send the packet safely.

\section{B. Anonymous Secure Routing in the Mobile Ad- Hoc Networks}

Zhu, Zhiguo Wan, Mohan S. Kankanhalli (2011) authors proposes the anonymous routing protocol [10]. Anonymity is a very important part of the overall solution for securing mobile ad-hoc networks. In this paper, we first gave a comprehensive definition on the goals that should be supported in anonymous routing protocols. To achieve them, we proposed the Anonymous Routing Protocol, which ensure both the anonymity and security of the routing protocol.

\section{Authenticated Routing For Ad Hoc Networks}

K. Sanzgiri et.al (2002) proposed the Authenticated Routing for Ad hoc Networks (ARAN) [11] protocol that uses public key cryptography instead of the shared security association. The intermediate node verifies the integrity of the received message before forwarding it to its neighbor nodes. The Source node and the destination nodes use certificates included in the route discovery and reply messages to authenticate each other. The cost of the certificates is high, limiting the ability of the attackers to request them limitlessly. The certificates can also help manage the network.

\section{ALERT: An Anonymous Location-Based Efficient Routing Protocol in MANETs}

HaiyingShen and Lianyu Zhao (2013) proposed the ALERT routing protocol [2]. Existing anonymity routing protocols in MANETs can be mainly classified into two categories: hop-byhop encryption and redundant traffic. The current approaches are limited, i.e. anonymity at a heavy cost to because public-key-based encryption and high traffic generate significantly high cost. Many approaches cannot provide all anonymity protections. The ALARM routing protocol cannot protect the location anonymity of the source node and destination node, the SDDR cannot provide anonymity for route, and ZAP provides only destination anonymity. There are many anonymity routing algorithms which are based on the geographic routing protocol that greedily forwards a packet to the node closest to the destination.

A packet in ALERT includes the source and destination zones rather than their positions to provide anonymity protection to the source and the destination. ALERT strengthens or tries to giveAnonymity protection to source and destination by hiding the data initiator/receiver among a number of data initiators/ receivers. It has the "notify and go" mechanism for source anonymity, and it also uses the local broadcasting method for destination anonymity. It also gives an efficient solution to counter intersection attacks. ALERT can also fight against timing attacks. However, the protocol's strict relay node selection makes it easy to reveal the source and destination and to analyze traffic.

E. ARM: Anonymous Routing Protocol for Mobile Ad hoc Networks

StefaanSeys and Bart Preneel, K.U.Leuven (2009) authors proposed the ARM routing 
protocol [12]. The main drawback of ASR and ANODR is efficiency:

1. Every forwarding node has to generate a fresh public/ secret key pair for every RREQ message it forwards. As these RREQs are flooded over the entire network, every node in the network needs to generate a fresh key pair for every RREQ that is released in the network. The private key is stored in the node's routing table. Note that the cost of generating public/private key pairs is non-negligible.

2. RREP messages carry no identifier that can be linked to the RREQ messages. This means that for a node to decide whether it has to forward a RREP or not, it has to try to decrypt it with every private key it has stored in its routing table.

3. The destination is identified using a trapdoor identifier. That indicates that "if you are the destination then you see this". This is the way the ARM routing protocol works with respect to anonymous routing communication for mobile adhoc networks.

\section{F. Mask}

Y. Zhang et.al (2005) says that anonymous authentication with low cryptographic overhead and high routing efficiency can be obtained by using proactive neighbor detection. This protocol is resistance to a wide range of adversarial attacks. MASK [9] is a proactive neighbor detection protocol to constantly see the snapshot of its one hop mobile neighborhood. The neighbor detection protocol is an identityfree detection protocol. Each node in MASK only knows the physical presence of neighboring nodes. This is achieved by a pairing-based anonymous handshake between any pair of neighboring nodes in a network.

MASK uses a three-way stage handshake for key exchanges among a node and its new neighboring nodes. In the handshake, each pair of nodes has to share a chain of secret key and locally unique Link Id pair. MASK does not use a global trapdoor.

In the MASK RREQ Packet, the source node puts in the destination node D's network ID and sends in a network. The processing overhead to open the global trapdoor is saved; end-to end key agreement is done and results in amore efficient RREQ procedure. Here the recipient anonymity is compromised by every RREQ receiver. Finally the routing information is not authenticated. So there is a chance that the data can be used by attacker.

\section{PROPOSED SYSTEM}

We propose a new routing protocol to overcome the pre-mentioned problems. We adopt onion protocol to record a discovered route and design an encrypted secret message to verify that if there is any linkage in route request or route reply or vice-versa.

Group signature is used to authenticate the RREQ packet for each node and also to prevent intermediate nodes from modifying the packet this group signature is available with all the nodes which are with in a particular group.

In the proposed system architecture which is shown in fig 1, the base station will generate the node pseudonyms (different name), group key and the public key and private keys for better security. Each node will store the group signature in the key store.

The pseudonyms will be stored in the pseudonyms store which is present in the each node. The route request and route reply generation and validation is also done by each individual node so that there is no overhead for the base station. After validating correct signature the data is transferred to the next node so on till it reaches the destination. The destination node also sends the reply through the same path that it has got the data to the source.

In this way the data is transferred safely and securely without any intruders involved. If in any case the intruder wants to involve and use the data it should have the group key and session key for that particular movement. This is impossible. So the data is transferred safely to the destination node.

The routing protocol used here is onion routing which protects the intermediates nodes from 
Asia Pacific Journals

inferring the destination and uses the route secret verification message by using pseudonyms. So that the intruder finds it difficult to identify which is the packet and what data it is sending. The main goal of this protocol is to make the packet safe from the intruder so that the packet reaches safely to the other end or the destination.

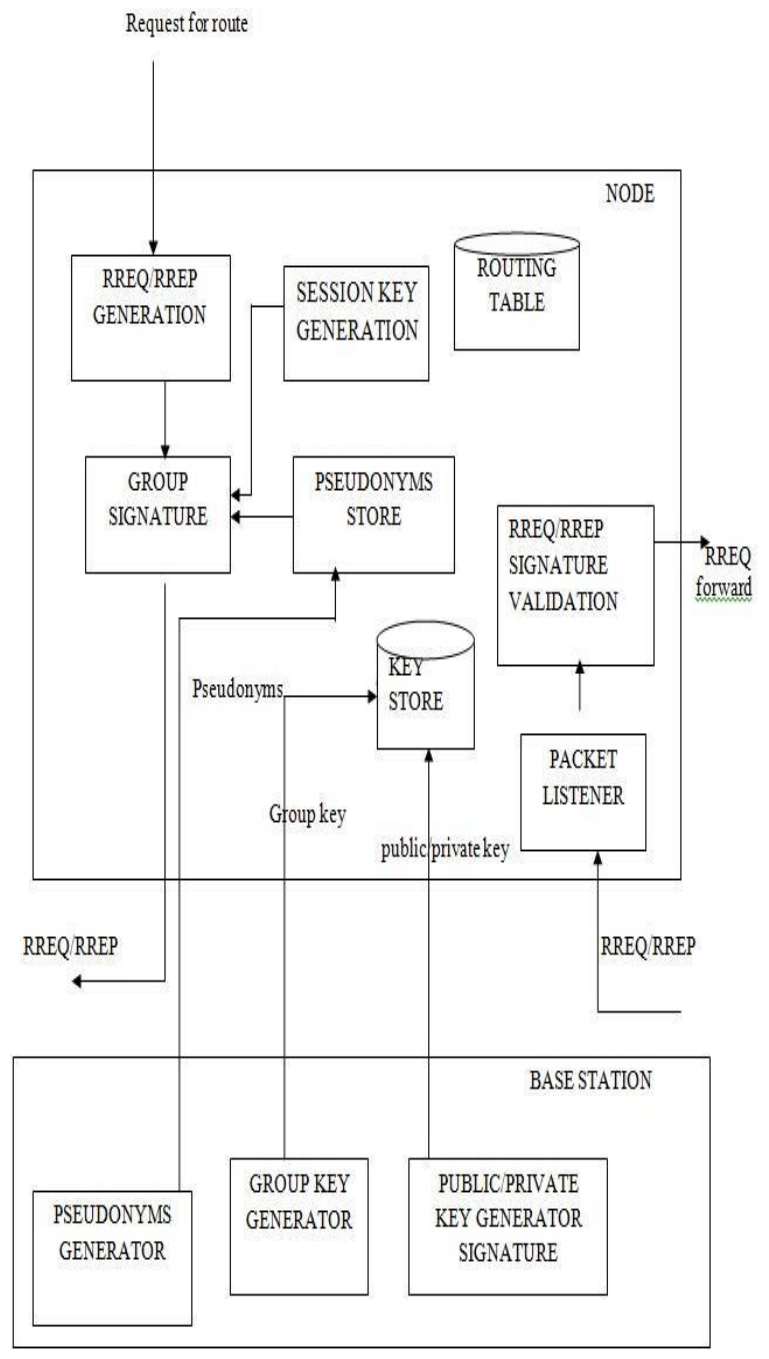

Fig1. System Architecture

The fig. 2 shows how the how the onion routing protocol works. The route request and route reply shown in the figure shows how the onion routing protocols works through which data is transferred. By using this method we can say the data will be sent to the destination safely and there will be no packet loss because the group signature, public key and private key shared between the nodes. The attacker cannot enter the medium because it is not having the group key. It also cannot see the data because it is encrypted using the onion routing protocol with public and private key. The onion routing protocol as the name specifies that each the data is secured with in the different layers as the layers of onion. Only the address of the next node is seen no data will be seen by the intermediate node, only the destination will see the data which is sent by the source to the destination.

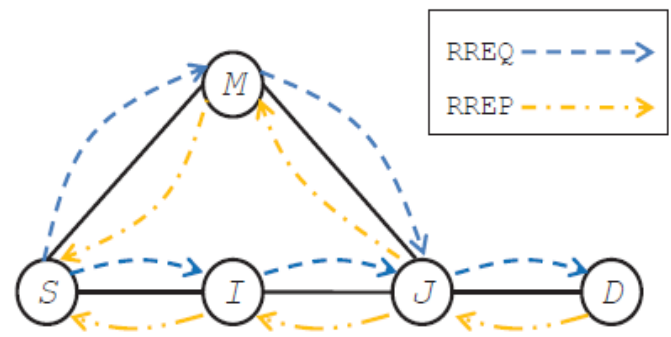

Fig2. Working of onion routing protocol

\section{PERFORMANCE SIMULATIONS}

We implemented the proposed protocol in java eclipse with the help of existing AODV routing protocol. We can compare the performance with respect to delay and throughput.

\section{Simulation Results}

In the simulation results in fig 2 we can see that the throughput of AASR routing protocol is higher than the AODV routing protocol. So by using the AASR protocol we can get high throughput.

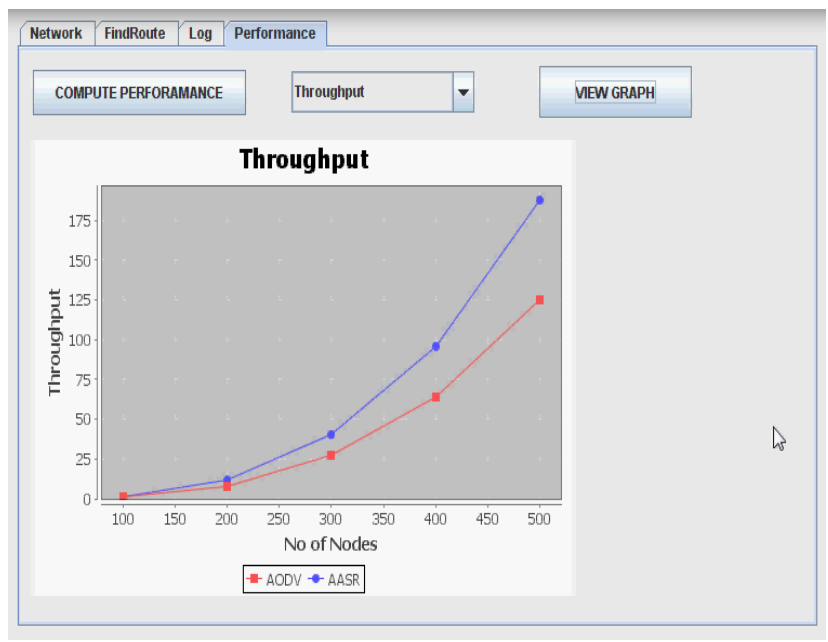

Fig2. No. of nodes vs. throughput 


\section{Asia Pacific Journals}

The second graph fig 3 illustrates that the delay caused due to flooding in AODV routing protocol is more compared to the AASR routing protocol. In AASR only one flooding takes place when the source needs to send the packet to the destination. After getting a reply from destination, the source sends the original packet along the shortest path which is got during flooding. But in AODV routing protocol the source send the request to the destination by flooding and again it sends the original data by using the flooding method in this method the delay to send and receive packet is more which can be seen in the graph.

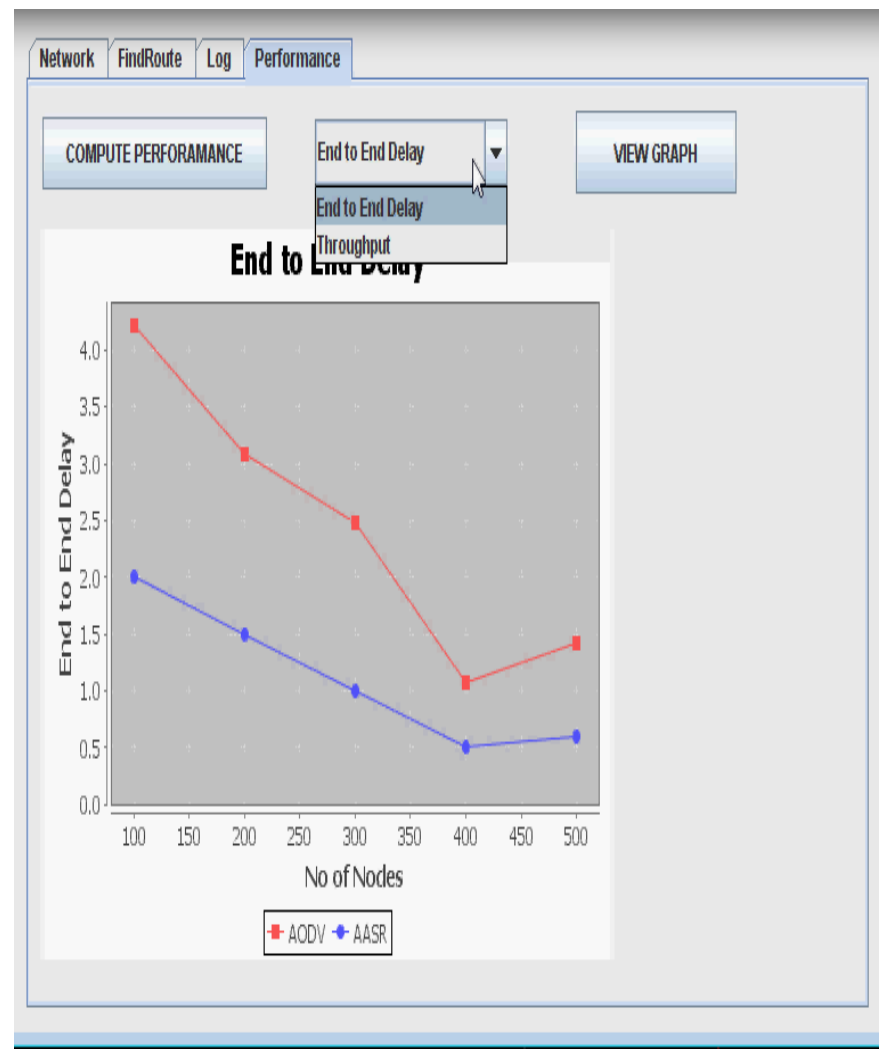

Fig3. No. of nodes vs. end to end delay

\section{CONCLUSION}

In this paper, we design an authenticated and anonymous routing protocol for MANETs in adversarial environments. The packets are authenticated by group signatures, which can defend the potential active the onion routing protocol with a route secret verification message is designed to not only record the anonymous routes but also prevent the intermediate nodes from inferring the real destination. The proposed system aims that with the help of the trust model, the routing protocols will be more active in detecting link failures caused either by the mobility or adversary attacks. So by implementing this protocol we can achieve greater security compared to AODV protocol.

\section{REFERENCES}

[1] StefaanSeys and Bart Preneel ,K.U.Leuven," ARM: Anonymous Routing Protocol for Mobile Ad hoc Networks" Int. Journal of Wireless and Mobile Computing, vol. 3,no. 3, pp. 145-155, Oct. 2009.

[2] Haiying Shen, and Lianyu Zhao, "ALERT: An Anonymous Location-Based Efficient Routing Protocol in MANETs", IEEE TRANSACTIONS ON MOBILE COMPUTING, VOL. 12, NO. 6, JUNE 2013

[3]P.Thamizharasi, D.Vinoth,"Unobservable Privacy-Preserving Routing In MANET", International Journal of Emerging Science and Engineering (IJESE) ISSN: 2319-6378, Volume-2, Issue-3, January 2013.

[4] Bo Zhu, Zhiguo Wan, Mohan S. Kankanhalli, Feng Bao, Robert H. Deng, "Anonymous Secure Routing in Mobile AdHocNetworks", International Symposium on DOI:10.1109/CNDS.2011.5764561 Publication Year: 2011 , Page(s): $142-147$

[5] K. E. Defrawy and G. Tsudik, "ALARM: Anonymous Location-Aided Routing in suspicious MANETs," IEEE Trans. on Mobile Computing, vol. 10, no. 9, pp. 1345-1358, Sept. 2011.

[6] J. Kong and X. Hong, "ANODR: Anonymous On Demand Routing with Untraceable Routes for Mobile Ad hoc networks," in Proc. ACM MobiHoc'03, Jun. 2003, pp. 291-302. 
[7] R. Song, L. Korba, and G. Yee, “AnonDSR: efficient anonymous dynamic source routing for mobile ad hoc networks," in Proc. ACM Workshop Security of Ad Hoc and Sensor Networks (SASN'05), Nov. 2005.

[8] J. Kong, X. Hong, and M. Gerla, “ANODR: An identity-free and on demand routing scheme against anonymity threats in mobile ad hoc networks," IEEE Trans. on Mobile Computing, vol. 6, no. 8, pp. 888-902, Aug. 2007.

[9] Y. Zhang, W. Liu, W. Lou, and Y. Fang, "MASK: Anonymous On demand Routing in Mobile Ad Hoc Networks," IEEE Transactions on Wireless Communications, no. 9, 2006.

[10] B. Zhu, Z. Wan, M. S. Kankanhalli, F. Bao, and R. H. Deng, "Anonymous Secure Routing in Mobile Ad-Hoc Networks," in LCN '04, 2004.

[11] Authenticated Routing For Ad Hoc Networks Sanzgiri, K. Dept. of Computer. Sci., Univ. of California, Santa Barbara, CA, USA LaFlamme, D. ; Dahill, B. ; Levine, B.N. ; Shields, C. ; Belding-Royer, E.M.

[12] ARM: Anonymous Routing Protocol for Mobile Ad hoc Networks Seys, S. ; Dept. of Electr. Eng., Katholieke Univ. Leuven; Preneel, B. Advanced Information Networking and Applications, 2006. AINA 2006. 20th International Conference on (Volume: 2) 